This item was submitted to Loughborough's Research Repository by the author.

Items in Figshare are protected by copyright, with all rights reserved, unless otherwise indicated.

\title{
Modelling infinite length panels using the finite element method
}

PLEASE CITE THE PUBLISHED VERSION

http://dx.doi.org/10.1142/S0219455417500389

\section{PUBLISHER}

(C) World Scientific Publishing

\section{VERSION}

AM (Accepted Manuscript)

\section{PUBLISHER STATEMENT}

This work is made available according to the conditions of the Creative Commons Attribution-NonCommercialNoDerivatives 4.0 International (CC BY-NC-ND 4.0) licence. Full details of this licence are available at: https://creativecommons.org/licenses/by-nc-nd/4.0/

\section{LICENCE}

CC BY-NC-ND 4.0

\section{REPOSITORY RECORD}

Fenner, Patrick, Andrew Watson, and C.A. Featherston. 2016. "Modelling Infinite Length Panels Using the Finite Element Method". figshare. https://hdl.handle.net/2134/21826. 


\title{
MODELLING INFINITE LENGTH PANELS USING THE FINITE ELEMENT METHOD
}

\author{
Patrick Fenner and Andrew Watson \\ Aeronautical and Automotive Engineering, Loughborough University \\ Leicestershire, LE11 3TU, UK \\ contact@def-proc.co.uk, A.Watson@lboro.ac.uk \\ Carol Featherston \\ Cardiff School of Engineering, Cardiff University, \\ Queen's Buildings, The Parade, Cardiff CF24 3AA, UK \\ FeatherstonCa@cardiff.ac.uk \\ Received (Day Month Year) \\ Accepted (Day Month Year)
}

\begin{abstract}
This paper compares three finite element models for determining the buckling and postbuckling performance of infinite length thin walled composite and metal stiffened panels - such as for modelling theoretical aircraft upper wing skin panels — single bay, double half-bay and quad half-bay models. The quad half bay model is shown to be the ideal model as all wavelengths of buckling are permitted. This model gives an accurate estimate of postbuckling behaviour that can include advanced behaviour such as mode jumping or collapse while the single bay and double half-bay models are more restrictive and do not allow for accurate mode jumping to take place. Sample panels are analysed for buckling performance using the computer program VICONOPT, which assumes an infinite length structure based on exact strip theory. This analysis is then compared to results from the quad half-bay FEM model, using the Abaqus solver, where the two models are in good agreement for the initial buckling performance for both the metal and composite panels. Bucking prediction for the quad half-bay model is within $0.5 \%$ for the critical buckling mode, and within $3 \%$ of all compared modes; and postbuckling performance compares well with the results of previous investigation of the same sample panel geometry.
\end{abstract}

Keywords: Panel Buckling; Postbuckling; Finite Element Method; FEM; Infinite Length; Finite Strip; FSM.

\section{Nomenclature}

$A=$ Cross sectional area.

$B=$ Panel width (equal to $b$ for a plate).

$D=$ Flexural rigidity of the plate.

$L=$ Length of mode.

$N_{x}=$ Area averaged, axial load per unit width.

$a=$ Plate length (equal to $l$ if panel is prismatic).

$b=$ Plate width (normally equal to stiffener pitch). 
$d=$ Axial panel compression (displacement)

$k=$ Buckling mode coefficient.

$l=$ Panel length.

$m=$ Number of longitudinal half wavelengths. $\left(m=l / \lambda_{x}\right)$

$n=$ Number of lateral half wavelengths. $(n=B / \epsilon)$

$q=$ Longitudinal wavelength series limit.

$t=$ Plate thickness.

$u=$ Displacement in the $x$-axis.

$v=$ Displacement in the $y$-axis.

$w=$ Displacement in the $z$-axis.

$\epsilon=$ Strain.

$\theta=$ Rotation about the $x$-axis.

$\lambda=$ Half-wavelength.

$\lambda_{x}=$ Longitudinal half-wavelength.

$\lambda_{y}=$ Transverse half-wavelength.

$\nu \quad=$ Poisson's Ratio.

$\xi \quad=$ Longitudinal wavelength quantifier.

$\sigma=$ Stress.

$\phi \quad=$ Rotation about the $y$-axis.

$\psi=$ Rotation about the $z$-axis.

${ }_{c r i t}=$ Critical condition (suffix).

All formulas and calculations use S.I. units exclusively.

\section{Introduction}

Modern aircraft wings are thin walled structures composed of ribs, spars and stiffened skins. The top skin is subject, under aerodynamic loading, to compressive forces that can cause buckling instability. As a simplification for analysis, the top wing surface can be divided into a series of rectangular, flat-skinned, stiffened panels with simply supported boundary conditions. Aircraft wing structure is also typically curved and tapered. So, while not all aircraft skin panels are such, this paper deals exclusively with panels that are flat and prismatic along the loading direction and looks at models for predicting their buckling and postbuckling behaviour. It is well known that flat rectangular plates, of the sort looked in this paper, behave in a stable manner such that the induced wave pattern will continue to take extra load in the post-buckling range. This fact has been utilised by designers of aircraft panels to reduce mass.

The nonlinear dynamics of plates has been well studied ${ }^{11} \mathrm{~A}$ complete and exact knowledge of plate statics or dynamics must contain a full three dimensional field which is often too difficult. Hence lower dimensional models are used for the type of elements used in this paper. For the analysis of plate structures, two common methods are, the Finite Strip Method (FSM) ${ }^{2}$ and the more general Finite Element 
Method (FEM) $!^{3}$ The critical bifurcation first emerges as a linear eigenvalue problem and this paper uses the industrially used linear elastic software VICONOPT, which is based upon exact finite strip theory ${ }^{4}$ and the commercially available FEM solver Abaqus to compute the eigenvalues.

The aim of this paper is to present a finite element model that can accurately determine the buckling and postbuckling response of an infinitely long panel, as described by finite strip theory. The FE model must be capable of finding all of the valid buckling modes for a finite strip equivalent structure, even if the modes are not found by any specific FSM method, but it must not introduce any erroneous modes that exist only as a result of the modelling method.

\subsection{Plate and Panel Buckling}

The Kirchoff-Love hypothesis assumes that the three dimensional plate can be represented by a two dimensional plane. When this hypothesis is applied to plates it leads to a fourth order partial differential equation governs the out-of-plane displacements which for a flat isotropic plate can be expressed as follows

$$
D \frac{\partial^{4} w}{\partial x^{4}}+2 D \frac{\partial^{4} w}{\partial x^{2} \partial y^{2}}+D \frac{\partial^{4} w}{\partial y^{4}}=N_{x} \frac{\partial^{2} w}{\partial x^{2}}+N_{x y} \frac{\partial^{2} w}{\partial x \partial y}+N_{y} \frac{\partial^{2} w}{\partial y^{2}}
$$

where $D$ represents the fleural rigidity of the plate and $N_{x}, N_{y}$ and $N_{x y}$ are longitudinal, transverse and shear loads per unit width.

Classical plate theory, which follows on from the Kirchoff-Love hypothesis, suggests that a plate will buckle into a mode shape dependant on both aspect ratio and applied load. $\frac{5}{5}$ Equation 1.2 defines the critical load per unit width for an axially applied loading on a simply supported plate (width $b$ and length $a$ ), resulting in a mode shape made up of a continuous pattern of an integer number of half sine waves (of length $\lambda$ ) in each orthogonal direction ( $m$ being the quantity running longitudinally and $n$ transversly).

$$
N_{x}=\frac{k \pi^{2} D}{b^{2}}
$$

where,

$$
D=\frac{E t^{3}}{12\left(1-\nu^{2}\right)}
$$

and

$$
k=\left(\frac{m b}{a}+\frac{n^{2} a}{m b}\right)
$$

Where $k$ is the buckling mode coefficient, which is dependent on buckling mode shape, and $t$ is the plate thickness.

Equation 1.4 shows that there is a set of buckling mode shapes from Equation 1.2 that could theoretically occur. It is the buckling mode that requires least load 
to occur that is critical. For critical values there is one half-wave transversely: $n=1$ - other loadings and boundary conditions will give different expressions for $k$. For stable postbuckling behaviour the opportunity may exist for the mode shape to jump to a different mode at a higher applied load. This is typically shown with an increase by unity of the number of half waves in the longitudinal direction, with $m$ changing to $m+1$.

A stiffened panel is a system of rigidly connected plates, consisting of a flat skin plate along with a number of stiffeners attached to it; where each can be a system of thin plates. The stiffeners effectively divide the skin plate into a series of flat strips. A selection of typical cross sections is shown in Figure 1 . The buckling modes of stiffened panels fall into three broad categories, local, torsional and overall (or global) modes for which the distinguishing features can be seen in Figure2, The panel cross section used in Figure 2 is that shown in Figure 1 a.

Stiffened panels can have a considerable postbuckling reserve of strength, enabling them to remain in stable equilibrium under loads in excess of their critical buckling load, when initial buckling is in a skin initiated local mode. This is when the inter-stiffener portion of skin (or stiffener pitch) that has the lowest critical buckling load initiates local buckling and deforms into its buckled shape. That deformation is propagated around the panel, both longitudinally and transversely, to form the buckled mode shape across the whole panel. Under further loading, other types of buckling can be observed such as overall buckling and torsional buckling; although, because of the complexity of this process, it is normal to use a computer program to predict this occurrence.

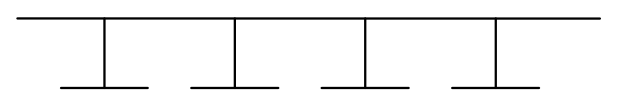

a)

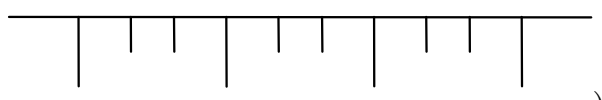

c)

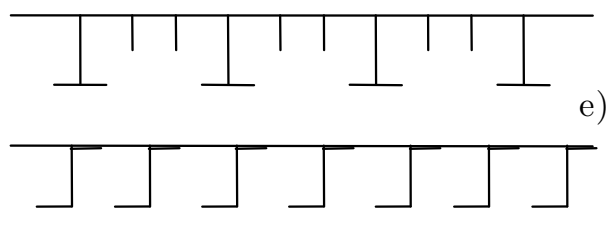

g)

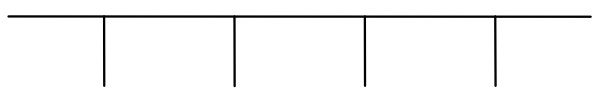

b)

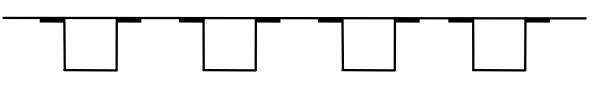

d)

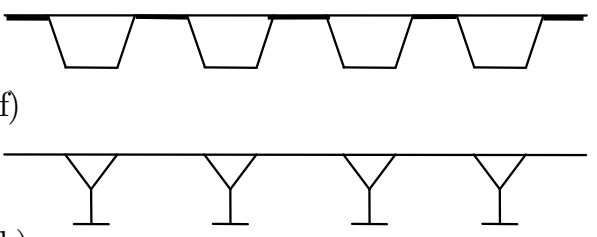

h)

Fig. 1: Thin-walled panel cross-sections.

An overall buckling mode typically shows skin displacements at all unsupported points on the skin. All the stiffeners displace in the plane of the stiffener web so all the line junctions between the stiffener web/skin and the stiffener web/stiffener 
flange displace out-of-plane relative to the skin. This is similar to plate buckling and the panel behaves as if the stiffener area is averaged over the width of the panel skin.

In an overall mode, the displacement of the stiffener web in its own plane leads to a significant reduction in overall stiffness; so these modes generally have poor postbuckling performance and as such, lead directly to panel failure and collapse. This mode type is characterised by a buckling mode shape that has one half-wave across the panel width as can be seen in Figure2a This is analogous to the buckling of a flat plate with $n=1$ in Equation 1.2 .

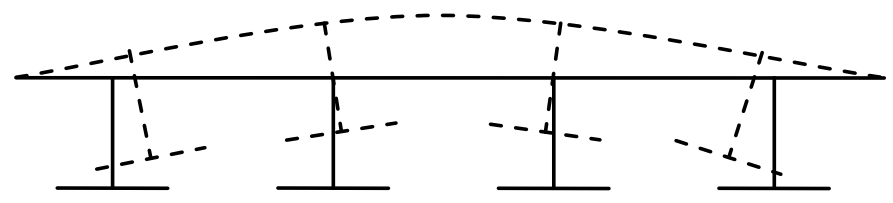

(a) Overall Buckling Mode

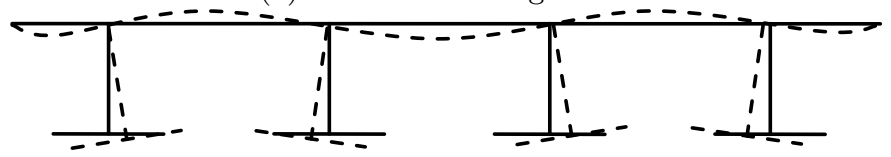

(b) Torsional Buckling Mode

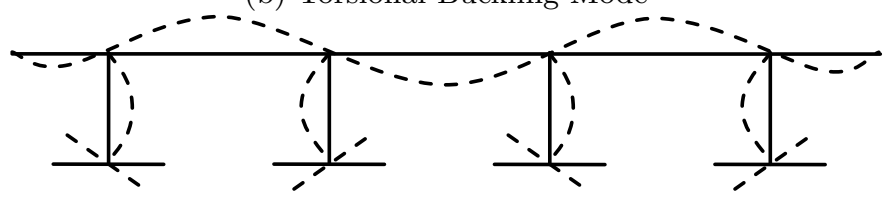

(c) Local Buckling Mode

Fig. 2: Buckling mode shapes of a typical stiffened panel.

Torsional buckling modes are characterised by small skin displacement, with skin/stiffener web line junctions remaining approximately straight and stiffener web/flange line junctions displacing in the plane of the stiffener flange. Figure $2 \mathrm{~b}$ shows significant displacement of the stiffener web/flange line junction relative to the skin/web line junction. These buckling modes generally have poor postbuckling performance because of the displacement of the stiffener, but can have a positive postbuckled stiffness in some circumstances. ${ }^{6}$

Local buckling modes show no displacement of the skin/stiffener and stiffener web/flange line junctions along the length of the panel to first order accuracy. All the displacement occurs between line junctions, with only rotation at the line junctions so using the skin pitch as the parameter $b$ in Equation 1.2 leads to a good approximation for the critical buckling load of the panel. Because the line junctions remain straight, load is transferred after buckling to these areas, allowing a significant postbuckling reserve of strength and positive postbuckled stiffness. 
Unlike buckling in columns, the initial buckling load of a panel can be different from the collapse load. When the initial buckling mode has a stable post-buckling stiffness, the load can be increased and the panel may "jump" into lower energy modes ${ }^{[7}$ where the stiffness for each mode will be less than the one preceding it, until the panel reaches an unstable buckling mode and collapses or the plate experiences a material strength limit.

\subsection{Modelling Buckling Modes}

VICONOPT's exact finite strip theory assumes a continuous distribution of mass and stiffness to produce a mathematically exact result for any longitudinally invariant plate structure that can be described by a number of rectangular cross section plates. This application of exact strip theory assumes that the buckling mode of a plate structure can be described by a sinusoidal response in an infinitely long plate, where this is a sum of specific half-wavelengths that factor exactly to the described panel length and are a function of the applied loading force.

VICONOPT $^{8}$ (VIPASA with CONstraints and OPTimisation) is a FORTRAN 77 computer program which incorporates the earlier programs VIPASA ${ }^{9}$ (Vibration and Instability of Plate Assemblies including Shear and Anisotropy) and VICON $\sqrt{10}$ (VIPASA with CONstraints). VICONOPT uses a dynamic stiffness matrix approach based on exact solutions to the governing differential equations and covers any prismatic assembly of isotropic, orthotropic or anisotropic plates, each of which can carry any combination of longitudinally invariant in-plane longitudinal, transverse and shear stresses. It can be used as either an analysis or an optimum design program.

The VIPASA and VICON analysis options of VICONOPT can both used to calculate the eigenvalues; which are either the critical load factors for buckling problems, or the natural frequencies in undamped vibration problems. The VICON option can be used to solve any analysis problem that could otherwise be solved by VIPASA, but has substantial additional capability because it models end conditions much more accurately for overall modes of plate assemblies with substantial in-plane shear loads, for which VIPASA gives very conservative results. This is because VIPASA assumes that the modes of buckling or vibration have a sinusoidal longitudinal variation with half-wavelength $\lambda_{x}$, whereas VICON modes are sums of such VIPASA modes obtained by coupling different values of $\lambda_{x}$.

The VICON option of VICONOPT assumes an infinitely long plate assembly and uses Lagrangian multipliers 11 to couple the responses for an appropriate set of half-wavelengths $\lambda_{m}$, so as to satisfy point support conditions at the panel ends, edges or interior of the panel that repeat at intervals of $l$. For example, a plate assembly of finite length $l$ with simply supported ends may be modelled by representing the simple support along the transverse line at $x=0$ by enforcing $w=0$ at a sufficient number of point supports at $x=0$ and this would also specify identical supports at $x=l$. 
The VICON results assume that the mode repeats over a length $L=2 l / \xi$ for some value $0 \leqslant \xi \leqslant 1$. Each value of $\xi$ generates an infinite series of $\lambda_{m}$, given by:

$$
\lambda_{m}=\frac{l}{\xi+2 m} \quad(m=0,1,2,3,4, \ldots)
$$

so that $\xi=0$ modes are identical in adjacent longitudinal bays, whereas $\xi=1$ modes are reversed and repeat every two bays. In practice the series is truncated by choosing a value of $q$ such that acceptable results are obtained by considering only the $\lambda_{m}$ for which, in Equation $1.5 .0 \leqslant m<q$ if $\xi=0$ or $\xi=1$, and $-q \leqslant m \leqslant q$ if $0<\xi<1$.

Negative values of $\lambda_{m}$ denote the use of complex conjugate stiffness matrices 10 in order to reverse the direction of the response. Table 1 lists the values of $\lambda_{m}$ derived from Equation 1.5 for typical values of $\xi$, it being noted that the analysis for negative values of $m$ is omitted for $\xi=0$ and $\xi=1$ because the response for any negative half-wavelength $-\lambda_{m}$ can be deduced from that for half-wavelength $\lambda_{m}$. The resulting transcendental eigenproblem requires an iterative solution which is performed using the Wittrick-Williams algorithm. 12

Table 1: Half-wavelengths $\lambda_{m}$ used in VICON analysis of a plate assembly of length $l$ with $q=3$.

\begin{tabular}{cc}
\hline$\xi$ & $\lambda_{m}$ \\
\hline 0 & $\infty, l / 2, l / 4$ \\
0.25 & $4 l,-4 l / 7,4 l / 9,-4 l / 15,4 l / 17,-4 l / 23,4 l / 25$ \\
0.5 & $2 l,-2 l / 3,2 l / 5,-2 l / 7,2 l / 9,-2 l / 11,2 l / 13$ \\
0.75 & $4 l / 3,-4 l / 5,4 l / 11,-4 l / 13,4 l / 19,-4 l / 21,4 l / 27$ \\
1 & $l, l / 3, l / 5$ \\
\hline Note: & $\lambda_{m}=\infty$ denotes rigid body displacement.
\end{tabular}

To validate any optimised panels designed using VICONOPT, the FEM model has to be able to model the initial buckling, postbuckling and collapse of a theoretical, infinite length, simply supported panel, equivalent to the conditions that VICONOPT assumes. Any validation model must be able to recreate any buckling mode that could occur in the theoretical structure, without introducing erroneous modes that occur only as a result of the modelling method.

Using Abaqus, $\frac{13}{13}$ it is straight forward to meet these requirements for a plate. In a 2D shell model, simple supports can be applied to each edge and load or displacement applied to the axial ends as shown in Figure 3 Only the loading method needs particular attention. Before initial buckling, load is applied with constant stress across the area, but in the postbuckled region, to keep the panel ends linear (and adhere to the conditions used by classical plate theory) the stress is re-distributed away from regions with large out-of-plane deflection. 


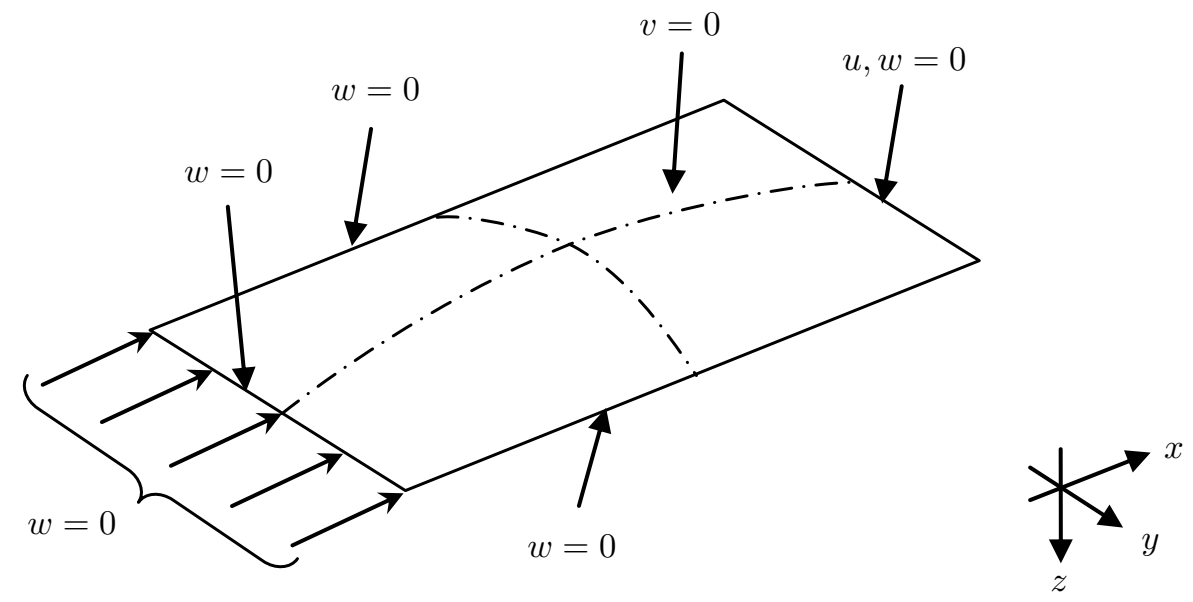

Fig. 3: Boundary conditions of a flat plate.

At the $y$-position where out-of-plane deflection is greatest along the length, stress in the $x$-direction is reduced (and may eventually become reversed); the load that would be carried by this area is distributed towards the places where there is less out-of-plane deflection, such as the lateral edges (for panels in local buckling modes, load is transferred towards the line junctions).

To remove the requirement to adjust the applied edge load, manually force is applied by controlling displacement. One end of the plate model has a fixed, zero axial displacement and an amount of axial displacement is applied to the opposite end, so the stress will vary automatically in post buckling.

The distribution of axial stress $\left(\sigma_{x}\right)$ at the displaced end is shown in Figure 4 , for a flat plate, for selected $d / d_{c r i t}$ levels. $d / d_{c r i t}=1$ denotes the stress distribution at the onset of buckling. The subsequent values of $d / d_{c r i t}=2$ and 3 show the increasing stress distributes towards the plate edges and a decrease of stress in the central portions of the plate.

The calculated results are for a $1 \mathrm{~m}$ square plate with $b / t$ ratio of 100 , made of aluminium with a Young's Modulus of 72.4GPa, and a Poisson's Ratio of 0.3. All Abaqus results presented in this paper use S4R elements. The S4R is a 4 noded linear shell element with reduced integration having one central integration point and default hourglass control. It is a general purpose conventional shell element which operates as a discrete Kirchhoff thin shell element when thickness is less than $1 / 15$ of the characteristic length on the shell surface as is the case here. The Kirchoff constraint is imposed numerically. These elements are finite strain elements which account for finite membrane strains and arbitrarily large rotations and are therefore suitable for large-strain analysis. 


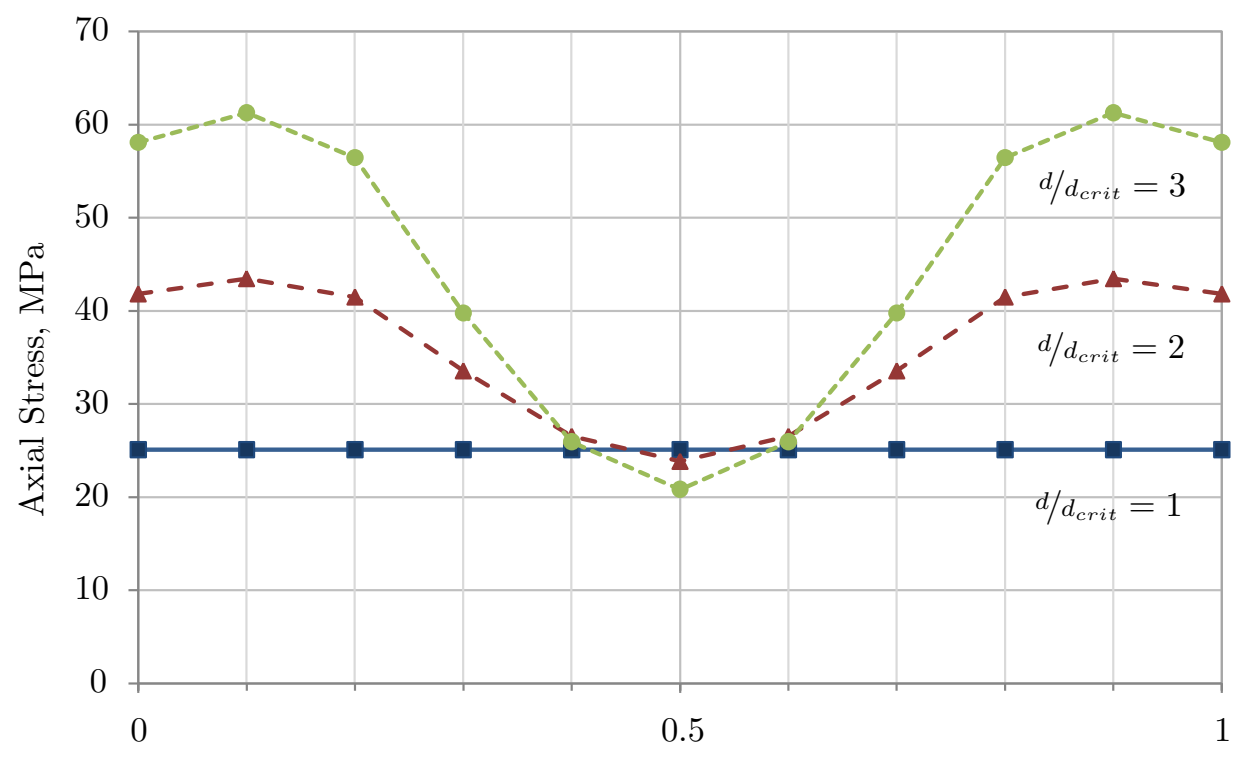

End Node $y$-position, normalised to width

Fig. 4: Postbuckling axial stress distribution for multiples of critical displacement.

\section{Modelling Panel Buckling}

To model panel buckling of an infinitely long panel in FEM, this section discusses the use of three models: the Single Bay Model, the Double Half-Bay Model and the Quad Half-Bay model. The precise details of the stiffened panels analysed are given in Section 3 below. They are taken from Stroud, Greene and Anderson 14 and both stiffened panels have the same topology, which is made up of a flat skin with six equally spaced blade stiffeners.

The infinite length model is more difficult to construct for a panel than a plate because the addition of stiffeners means the panel boundaries are now two dimensional and this makes the support conditions non-trivial. Modelling simple supports at these boundaries, although a reasonable approximation for a finite length panel, is not valid for an infinitely long panel. The boundary conditions and loading need to account for the fact that the cross section rotates and warps at the support as well as the stiffeners being continuous over at the boundary for the infinite length model. A mesh sensitivity analysis was carried out to ensure converged numerical results. This resulted in the use of at least ten elements per half-wavelength.

\subsection{Single Bay Model}

The starting point for panel modelling is a single bay model. As for the case of the plate model (Figure 3), the skin is simply supported on all edges i.e. at $x=0, l$ and $y=0, B$. At these lines, out of plane displacement is constrained such that $w=0$ 
and lateral displacement is constrained along the centline at $y=B / 2$ with $v=0$. Axial loading and displacement constraints, though, need particular attention.

As described above, applying displacement or load at a line away from the axial centroid will induce a bending moment to the panel; so for accuracy, this must be applied at, or averaged across, the centroid of area. The panel cannot be loaded at each end with constant stress because, as with the plate model discussed in Section 1.2. stress will be redistributed across the component plates based on the buckling mode. Since the aim of the analysis is to determine the postbuckling mode and performance, this cannot be known beforehand.

Displacement control could be applied to the end section, but not without some caveats. If displacement is applied only to the axial centroid, this will cause a stress concentration around this line. This will cause a non-uniform stress distribution before buckling that cannot redistribute over the cross section after buckling. The result of this loading case would show a length at each end of the panel where the stress distribution through the material is not representative of the infinite length case.

To allow the correct distribution of stress, a constant displacement could be applied to the whole cross section, as in the plate example above and shown for a six bladed stiffened panel in Figure 5 This is representative of "diaphragm end conditions" as described by Dawe and Wang ${ }^{15}$ where in the plate's local displacements at $x=0, u=d$; at $x=l, u=0$; and at $x=0, l, w=0$ and $v \neq 0$; or "component plates are simply supported for out of plane behaviour and are free to expand in their planes"
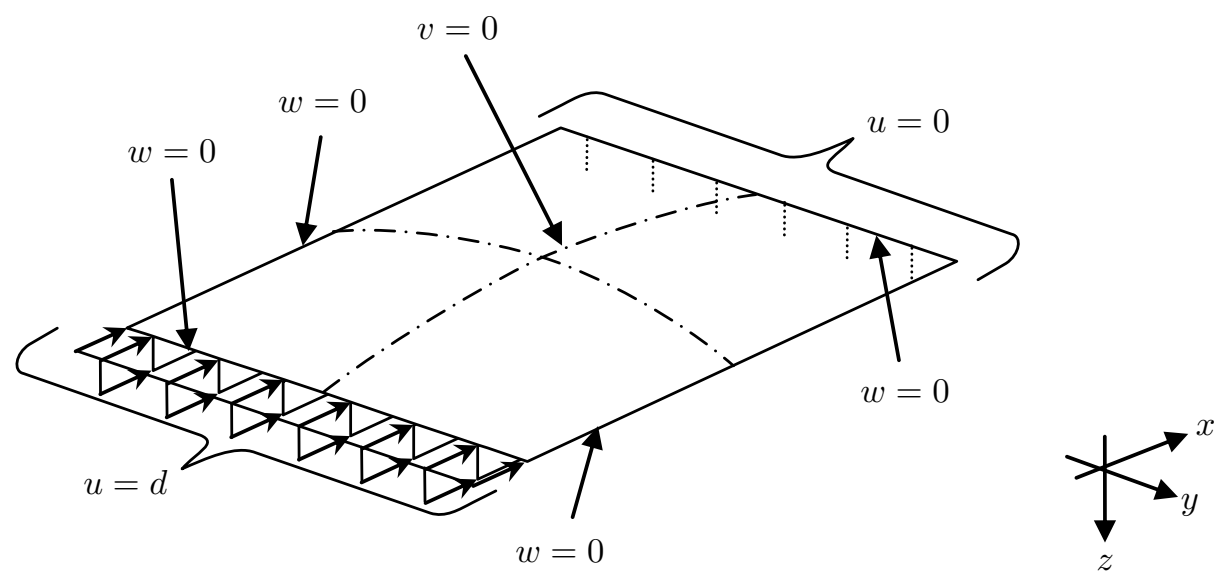

Fig. 5: Single bay model boundary conditions.

While a single bay model is sufficient for most panel buckling studies, using the uniform displacement method is unsuitable for modelling infinite length panels because it does not allow for cross section rotation at the panel ends and so cannot 
allow for all possible buckling mode shapes e.g. overall modes (see Figure 6). For local (and torsional) buckling modes the panel cross section does not warp or rotate significantly about the transverse axis ( $y$ axis) and so the application of constant displacement will correctly identify local buckling behaviour.

For overall modes in panels, the skin deflects out-of-plane in all locations except at the nodal lines because the skin plates are constrained to $w=0$ at $x=0, l$ and at $y=0, B$. However, at the nodal lines, the stiffeners will rotate about the $y$-axis, and this rotation will be the same as the $y$-axis rotation of the adjoining skin in the next single length bay.

For overall modes, the rotation of each stiffener is dependent on how much each stiffener bends during the mode. This results in the rotation, at the supports, of the central two stiffeners being greater than the rotation of the outer stiffeners, as seen in Figure 6. This means that while the cross section scales for Poisson's effect, it also warps and becomes non-planar. As such, the single bay constant displacement procedure will not correctly predict the simply supported response for overall modes and would incorrectly predict a higher-load mode to be critical.
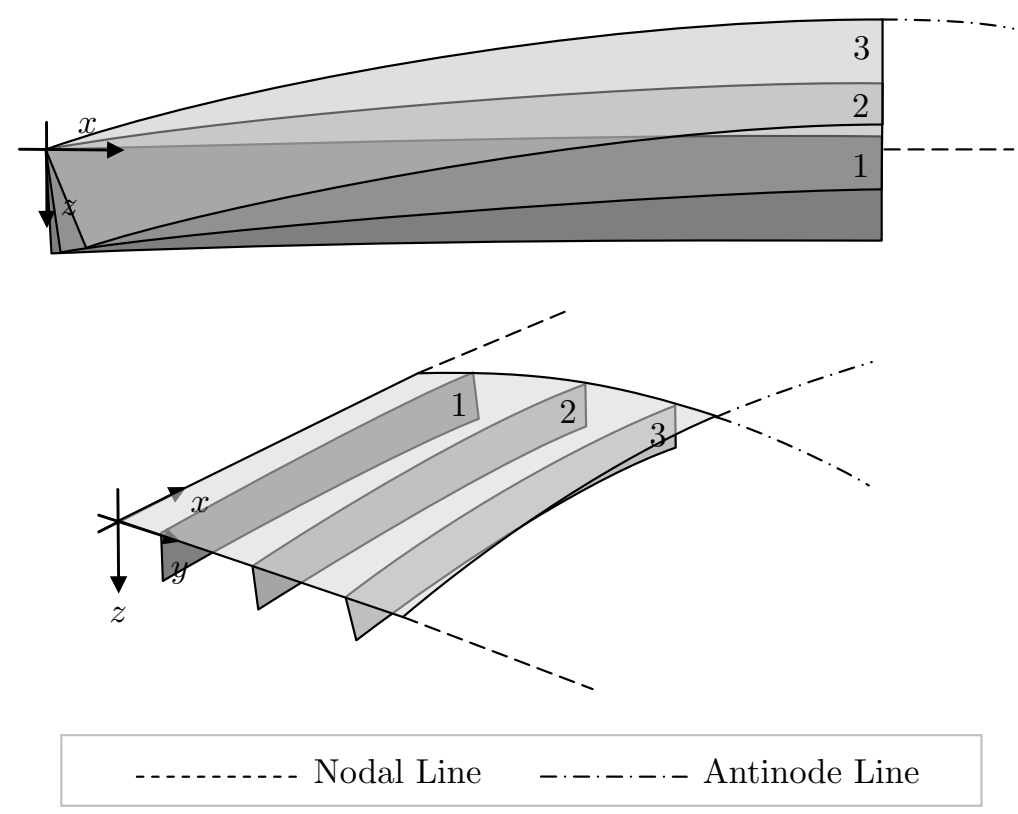

Fig. 6: End rotation of stiffeners for and overall mode.

\subsection{Double Half-Bay Model}

For axially loaded, infinitely long panels there is modal repetition along the length of the panel and therefore the mode in an infinite length panel must be symmetric 
about some $x y$-plane. As such, it must be possible to load the panel with constant displacement on that plane. Because the buckling behaviour predicted by VICONOPT assumes that the buckling modes will have a sinusoidal pattern they will therefore be symmetric about any antinode, shown in Figure 6.

The addition of stiffeners does not affect this plane of symmetry, meaning that it is possible to load a stiffened panel with constant displacement at a known antinode position but because overall modes do not have a positive postbuckling stiffness, only one antinode position need be considered: that of the critical overall mode. For the tested panel, as with many panel structures, the overall mode with the lowest load is the $\lambda=l / 1$ mode, so the position of the antinode will be at $x=l / 2$, so the panel can be modelled by two half-length panels joined at the transverse support and displacement loaded at the end planes as shown in Figure 7

For panels where the critical overall mode is $\lambda=l / 1$, the loaded end crosssections will at an antinode position for the critical overall mode and all other overall modes will not occur in practice. For the local and torsional modes the loaded end cross section will be at either a node or antinode position that both, as explained earlier, remain planar before and after buckling. While loading position will still not identify overall buckling modes where $m$ is even, these will all be at a higher buckling load than the critical overall buckling load (the upper bound for the collapse mode), and therefore will occur neither as an initial mode nor as a postbuckling mode.
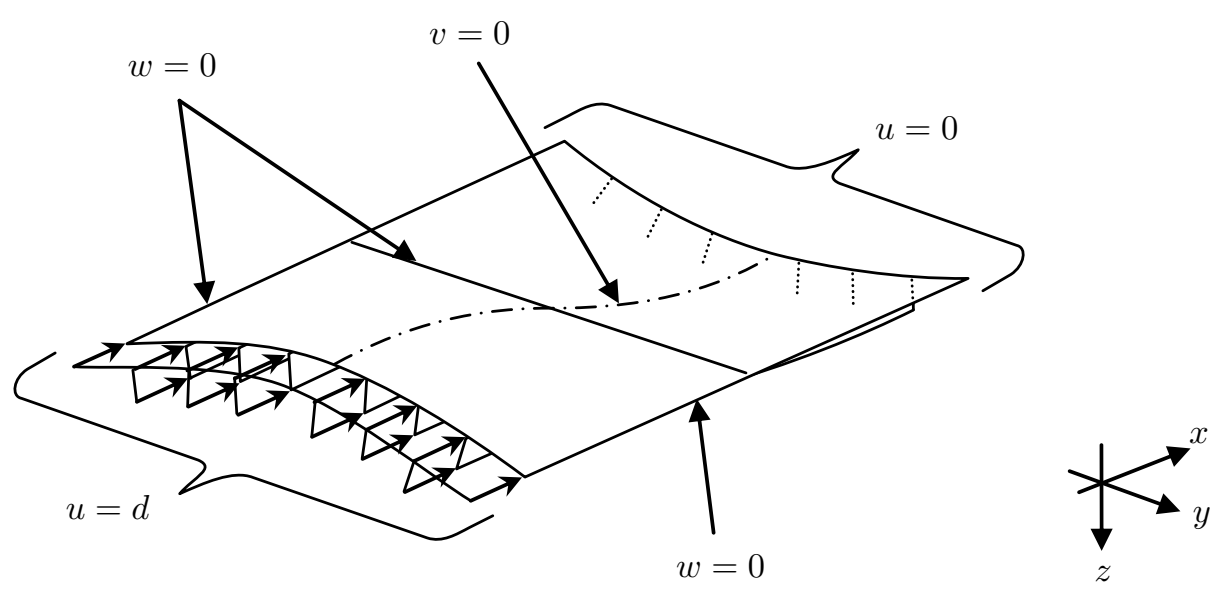

Fig. 7: Double half-bay model and boundary conditions.

For every mode with an odd number of half-wavelengths, the panel mode is reversed along each panel length; but in modes where $m$ is even, the mode repeats for every panel length. Figure 8 shows the comparison between the odd and even mode shapes. To prevent buckling mode shapes with discontinuous response at the 
model ends, some linking must be made between the angle and displacement of equivalent modes at each end of the FEM model.

The displacement relationships that must be applied to the displaced ends of the double half-bay model can be described by the following equations for each equivalent pair of end nodes (at $x=0$ and $x=l$ ).

For odd modes $(m=1,3,5, \ldots)$ :

$$
v_{0}=-v_{l}, \quad w_{0}=-w_{l}, \quad \theta_{0}=-\theta_{l}, \quad \phi_{0}=-\phi_{l}, \quad \psi_{0}=-\psi_{l}=0
$$

And for even modes $(m=2,4,6, \ldots)$ :

$$
v_{0}=v_{l}, \quad w_{0}=w_{l}, \quad \theta_{0}=\theta_{l}, \quad \phi_{0}=\phi_{l}, \quad \psi_{0}=\psi_{l}
$$

And $u_{0}=d, u_{l}=0$ for both odd and even modes.

As both sets of equations cannot be applied to the model at the same time, the double half-bay model is only capable of resolving either the odd, or the even number mode results. While running two simulations and concatenating the results might be suitable for buckling investigations; for modelling postbuckling, this separation of mode shapes prevents mode jumping between odd and even mode shapes where it could be a valid result.

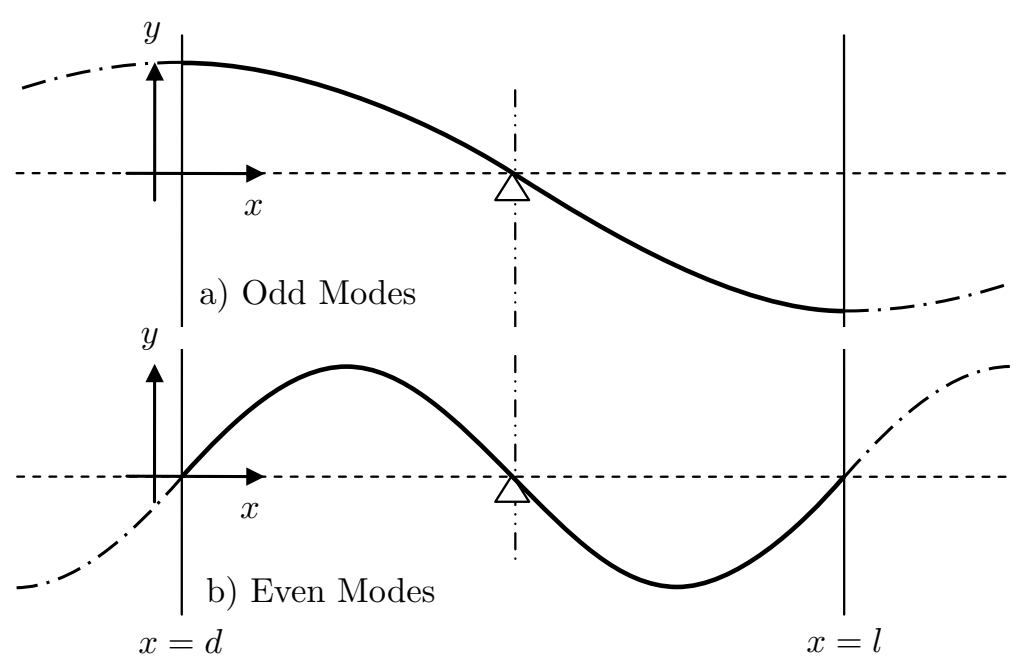

Fig. 8: Comparison of odd and even longitudinal modes.

The double half-bay model is essentially a pair of panels with one edge free and three edges simply supported, as shown in Figure 7 , loaded on the free edges. Plates that are loaded on an unsupported edge will have buckling modes that have large out of plane deflections at that edge, which would not occur in infinite length panels. Such an erroneous mode is shown in Figure 9 


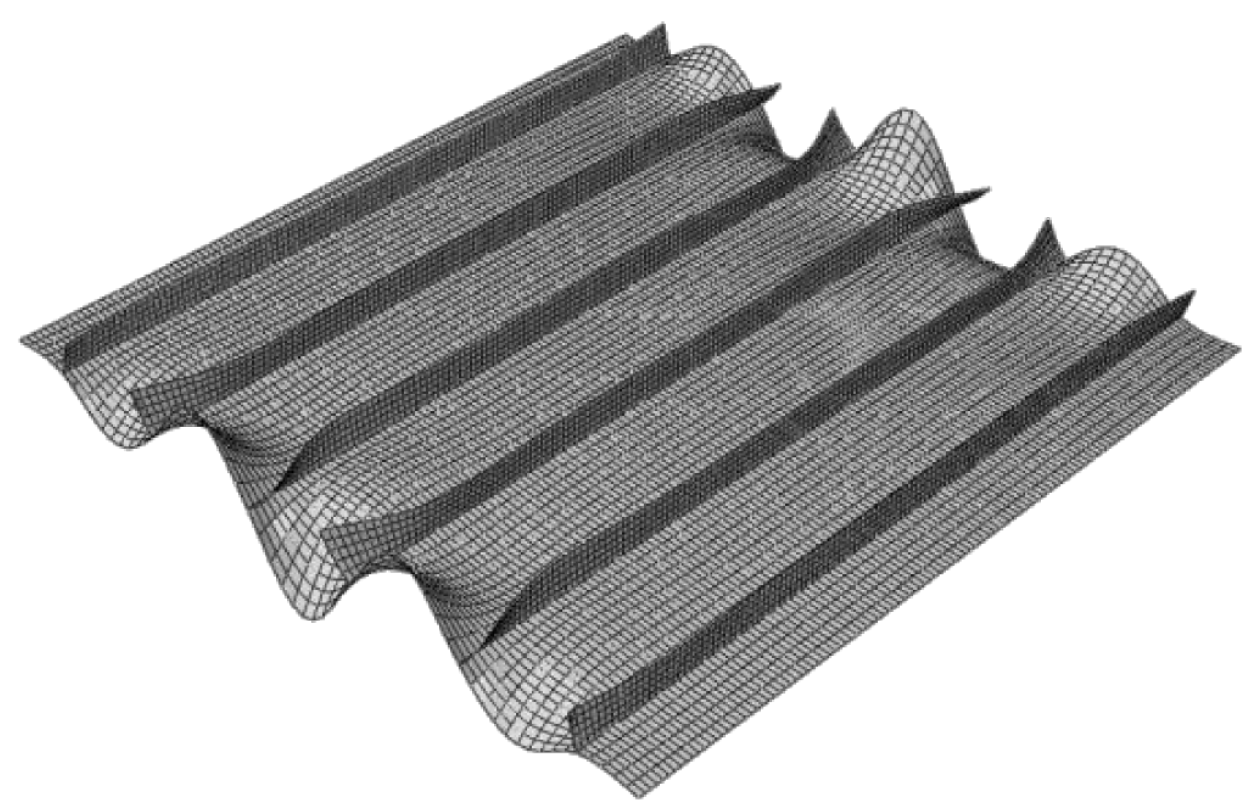

Fig. 9: Erroneous buckling mode shape for the double half-bay model showing end effects

\subsection{Quad Half-Bay Model}

To resolve the problem of mode separation, the length of the model can be doubled, as shown in Figure 10. For both odd and even modes there is repetition every four half-bays. This results in the following displacement relationships for each of node pair (at $x=0$ and $x=2 l$ )

For all modes (odd, $m=1,3,5, \ldots$ and even, $m=2,4,6, \ldots$ ):

$$
v_{0}=v_{2 l}, \quad w_{0}=w_{2 l}, \quad \theta_{0}=\theta_{2 l}, \quad \phi_{0}=\phi_{2 l}, \quad \psi_{0}=\psi_{2 l}
$$

And $u_{0}=2 d, u_{2 l}=0$ for both odd and even modes.

This model suffers from increased solution time as the number of elements is doubled, but accuracy is greatly increased over the double half-bay model by allowing correct solution of, and mode jumping between, all valid modes for the theoretical, infinite length panel. On the constrained transverse edges, where $w=0$, the stiffeners are free to rotate and the cross section is allowed to warp. Because displacement is applied and not load, no extra bending is introduced as a result of the shift in the neutral axis at and after initial buckling.

\section{Postbuckling Procedure}

The postbuckling procedure using an FEM solver is a two step process. The first step is to perform a buckling analysis of the panel from which a buckling mode shape 


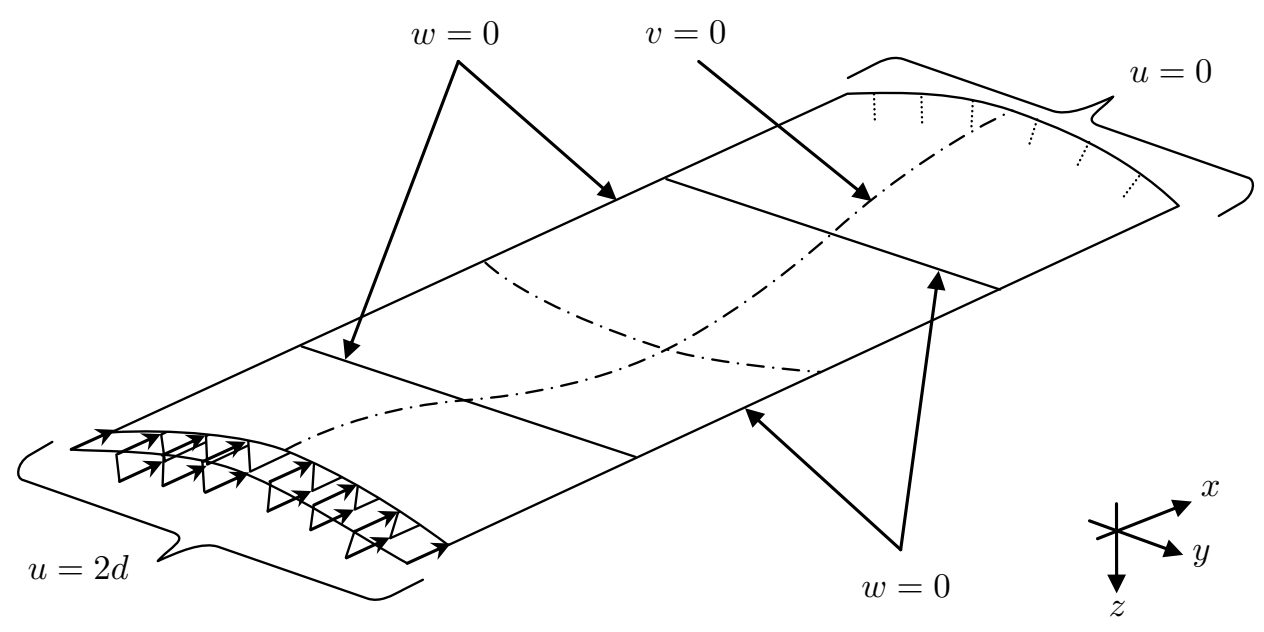

Fig. 10: Quad half-bay model and boundary conditions.

can be selected. The second step model is a general non-linear analysis, where the perfect model geometry is seeded with a small imperfection of the buckled mode shape obtained from the buckling analysis to allow for a post-buckling behaviour. For the first step, a buckling analysis model is created according to the boundary conditions shown in Figure 10. Incremental displacement control is applied in the second step, which allows for the postbuckling path to be tracked during both stable and unstable behaviour without needing to use a path following (Rik's) solver.

At the onset of collapse, which could be a mode jump from a local mode to an overall mode, there is significant load drop off. Numerical difficulties can also be encountered with solvers at a mode jump. When a mode jump occurs there is a step change in force and this discontinuity requires the solver to reduce incremental displacement step size. To allow the solver to continue past this point, a damping coefficient can be included in the solver to "smooth" the jump, though it should be noted that the minimum step time may still need to be reduced as well.

\section{Stiffened Panel Examples}

The stiffened panels used in the investigation are based on two examples taken from Stroud, Green and Anderson's paper, $\frac{14}{14}$ the "NASA Example 1" and "NASA Example 4" panels. The Example 1 panel is modelled with graphite-epoxy composite material and the Example 4 panel is modelled with aluminium. Both panels are identical in topology with a square skin of $762 \mathrm{~mm}$ and six equally spaced stiffeners as seen in Figure 11. The Example 4 panel has a skin thickness, $t_{s k}$, of $1.27 \mathrm{~mm}$ and a stiffener thickness, $t_{w}$, of $1.473 \mathrm{~mm}$. The aluminium has a Young's modulus of $72.4 \mathrm{GPa}$ and a Poisson's ratio of 0.32 . 


\subsection{NASA Example 4 Panel}

The metal panel has also been previously analysed by Dawe and Wang postbuckling performance was analysed using non-linear spline FSM; Peskham and Dawe ${ }^{16}$ where buckling and postbuckling performance was predicted using semi analytical FSM; and Stroud, Greene and Anderson ${ }^{14}$ who conducted an FEM study of performance, with all studies in close agreement of panel performance.

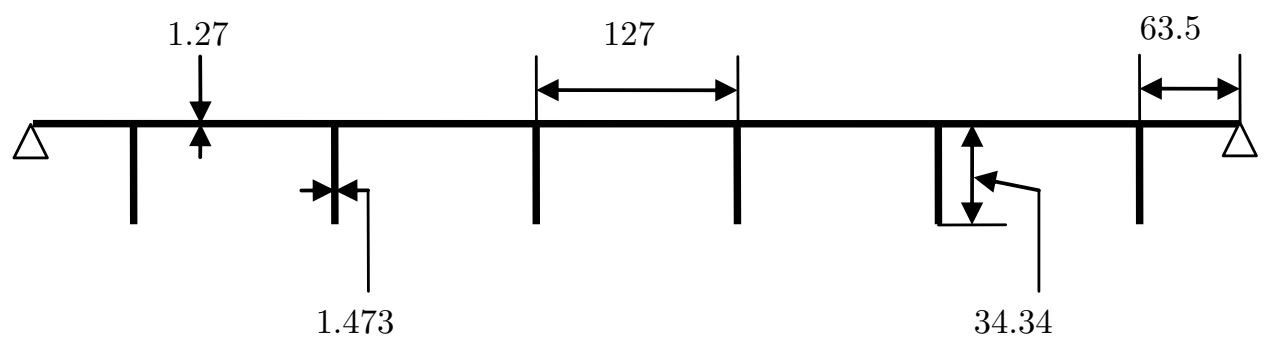

Fig. 11: Cross-section of "NASA Example 4" panel. Centreline dimensions in mm.

The quad half-bay model has been validated against both previously published results, and the authors' VICONOPT results. As explained in Dawe and Wang's paper ${ }^{15}$ it has been previously calculated that the effect of including the offsets ${ }^{9}$ between the web ends and skin centreline at the junctions has a negligible effect to the buckling characteristics, so for these results, and to allow direct comparison between current and previous results, the plates are joined at the centrelines.

A comparison of buckling modes and their buckling loads is presented in Table 2. with values given as multiples of the lowest buckling mode for the panel, the $\lambda_{x}=l / 6$ mode (shown in Figure 12 that has a critical load of $39.4747 \mathrm{kN}$ at an end displacement of $0.32766 \mathrm{~mm}(420 \mu \epsilon)$.

A visual inspection was required to identify the wavelength of each of the FEM mode shapes to compare the corresponding results to the VICONOPT results. Values are shown for the lowest eigenvalue of each mode shape, with all but the $m=1$ mode being a local type mode.

As the load factor comparisons show, for buckling, the Abaqus results are very close to the theoretical results from VICONOPT. Most are within $0.5 \%$ of the equivalent VICONOPT loads, but the greatest difference of magnitude is $2.34 \%$ which for the overall mode at a large eigen number is a reasonable result as the mode shape shows a superimposed transverse wavelength that the VIPASA model is unable to create.

The resultant modes show a good correlation between modelling methods. When compared by order of criticality (mode no.), the FEM analysis identifies many more modes than VICONOPT. The $\lambda_{x}=l / 1$ mode is the $85^{\text {th }}$ mode for VICONOPT, and $153^{\text {rd }}$ mode for the FEM model. The differences between the result sets are 


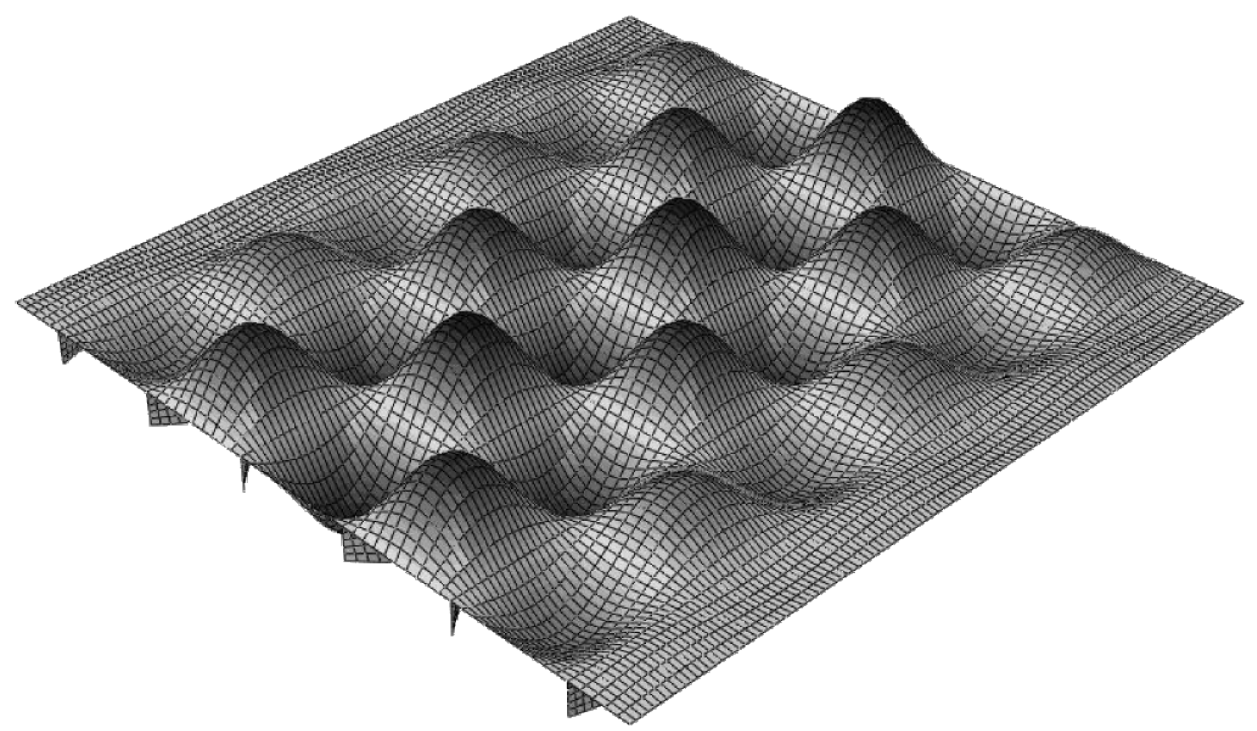

Fig. 12: Critial $(m=6)$ buckling mode of NASA Example 4 panel, single bay model.

Table 2: Comparison of Buckling Loads by Calculation Method.

\begin{tabular}{cccccc}
\hline $\begin{array}{c}\text { Mode No. } \\
m=\end{array}$ & $\begin{array}{c}\text { VIPASA } \\
\text { Order of } \\
\text { Criticality }\end{array}$ & $\begin{array}{c}\text { Buckling } \\
\text { Load Factor }\end{array}$ & $\begin{array}{c}\text { Order of } \\
\text { Criticality }\end{array}$ & $\begin{array}{c}\text { Abaqus } \\
\text { Buckling } \\
\text { Load Factor }\end{array}$ \\
\hline 6 & 1 & 1.0000 & 1 & 1.0002 & $(+0.02 \%)$ \\
7 & 2 & 1.0112 & 2 & 1.0120 & $(+0.08 \%)$ \\
5 & 3 & 1.0378 & 5 & 1.0386 & $(+0.08 \%)$ \\
4 & 9 & 1.1652 & 15 & 1.1683 & $(+0.264 \%)$ \\
3 & 35 & 1.5001 & 62 & 1.5077 & $(+0.51 \%)$ \\
2 & 76 & 2.4930 & 138 & 2.5306 & $(+1.51 \%)$ \\
1 & 85 & 2.8752 & 153 & 2.8079 & $(-2.34 \%)$ \\
\hline
\end{tabular}

explained by the assumption of sinusoidal variation along the length of the mode shape in VIPASA. The FEM model does not make any sinusoidal assumption that restricts the half-wave lengths to a constant amplitude along the length. Modulated wave forms are then allowed for in FEM model for which an example can be seen in Figure 13 .

The postbuckling behaviour of the quad half-bay FEM model shows very good agreement when compared to the results from Dawe, Lam and Azzizan. ${ }^{17}$ The stable part of the postbuckled load displacement plots of Figure 14 is almost identical to those published in the same paper, $\frac{17}{17}$ including the $l / 7$ mode having a higher stiffness than the $l / 6$ mode after buckling, as shown in detail in Figure 15 .

Both the $l / 6$ and $l / 7$ mode shapes were used as mesh seeds for separate analyses 


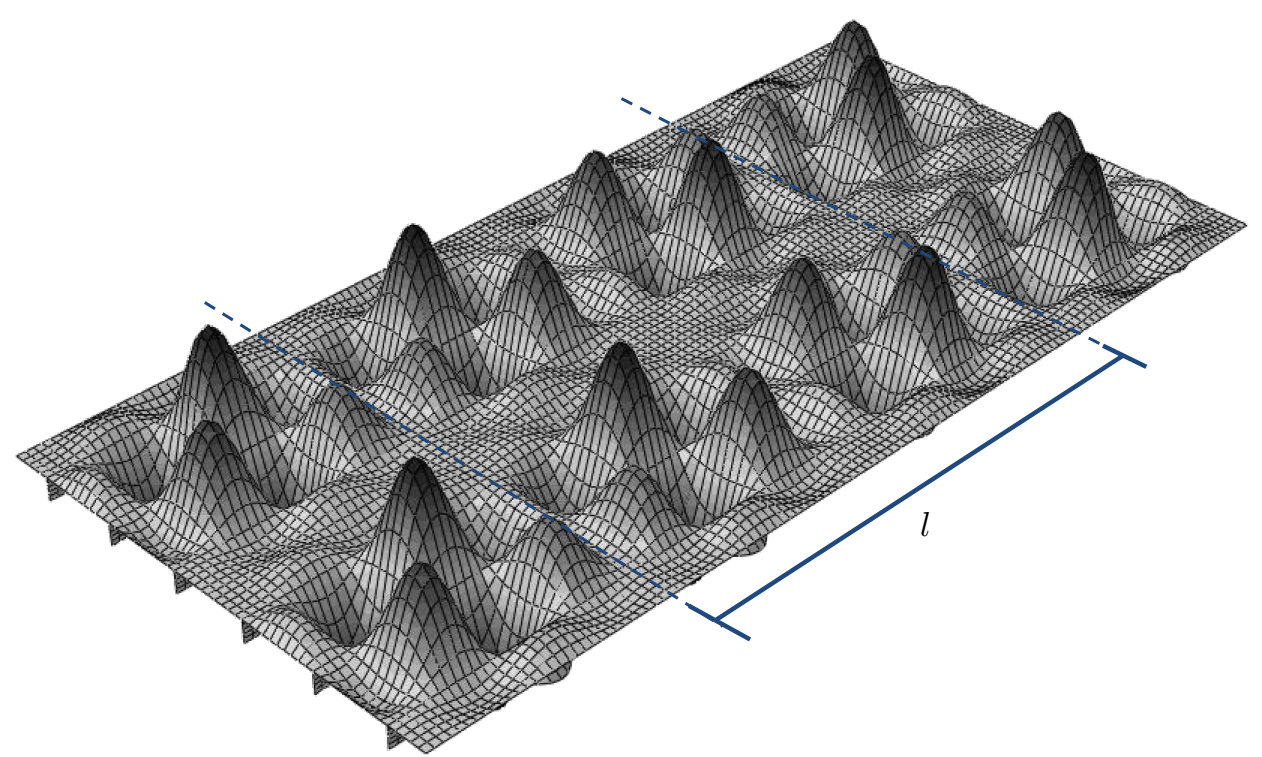

Fig. 13: Infinite plate buckling mode with modulated amplitude, from quad half-bay model (mode no. 17, load factor: 1.1850).

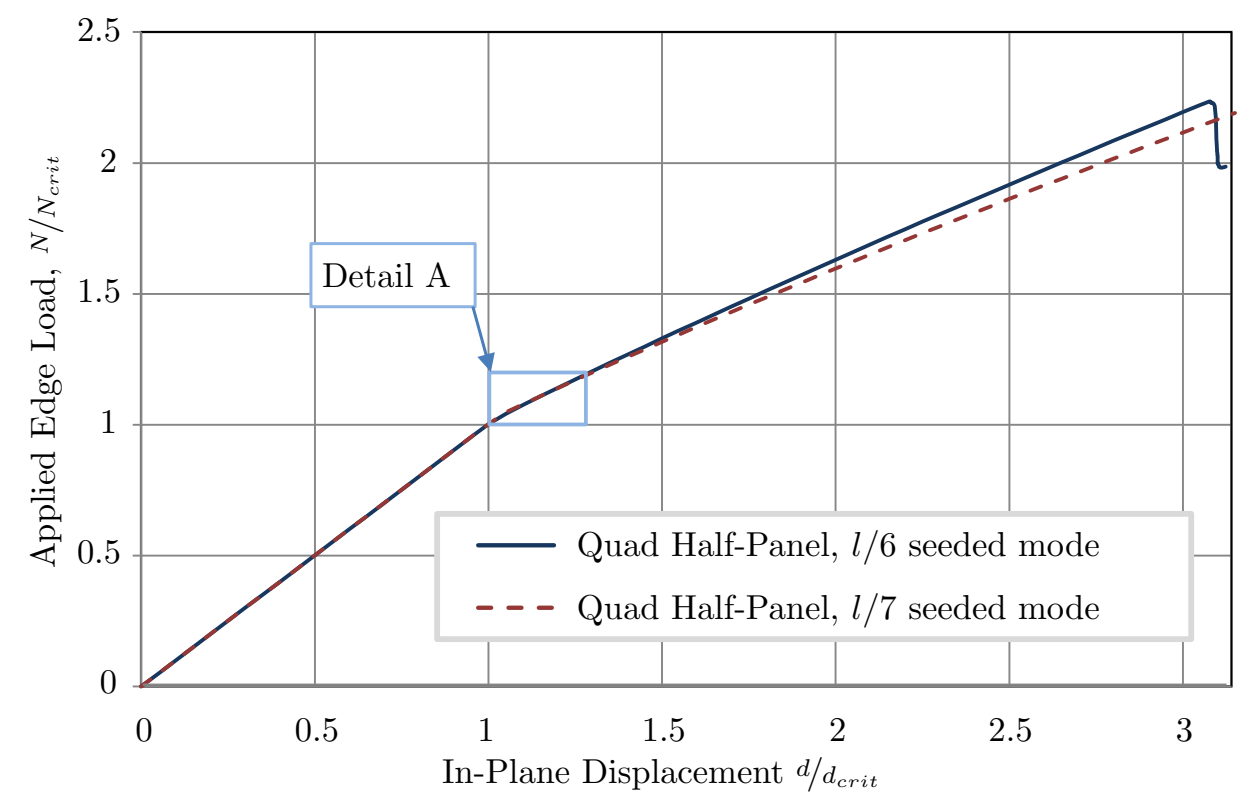

Fig. 14: Force vs. in-plane displacement from Abaqus quad half-bay postbuckling. 


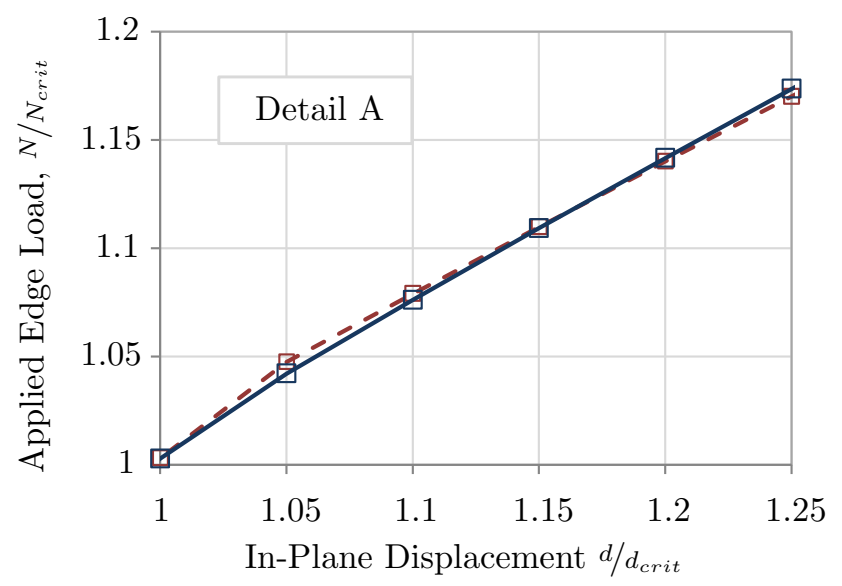

Fig. 15: Detail-A from Figure 14. The small squares indicate the load / displacement data points

using Abaqus but the results showed that the panel does not - as has been previously suggested - change into the lowest stiffness mode. For both these results, the panel remained in the seeded mode from initial buckling all the way through to collapse which was demonstrated as mode jumping into the overall mode, $\left(\lambda_{x}=l\right)$ without any intermediate changes.

For the $m=6$ seeded results, the panel jumped to the overall mode at 3.05 times critical strain and 2.23 times critical load as can be seen in Figure 14. The $m=7$ seeded panel was more stable, and entered the overall mode at 4.91 times critical strain and 3.01 times critical load.

\subsection{NASA Example 1 Panel}

This panel is a made from a composite layup of unidirectional graphite epoxy layers with a similar layout as the Example 4 panel, see Figure 16 . The original Example 1 panel, as modelled by Stroud, Greene and Anderson, $\frac{14}{14}$ exhibits critical buckling in an overall mode but, because overall buckling is a collapse mode, the panel is not well suited to testing postbuckling modelling with the original configuration. To adjust the buckling characteristics, an additional pair of layers (layer 6) were added to the stiffener layups to make them identical to the skin layup. Laminate layup is described in Table 3 , where only half the layers are explicitly defined for each plate element as they are symmetric.

The graphite epoxy has the following material properties: longitudinal Young's modulus $E_{x}=131 \mathrm{GPa}$; transverse Young's modulus $E_{y}=13 \mathrm{GPa}$; in-plane shear modulus $G=6.41 \mathrm{GPa}$; and Poisson's ratios $v_{x y}=0.38$ and $v_{y x}=0.0378$. While the original panel was tested either in axial shear, or in longitudinal compression, only longitudinal compression is tested in this paper. 


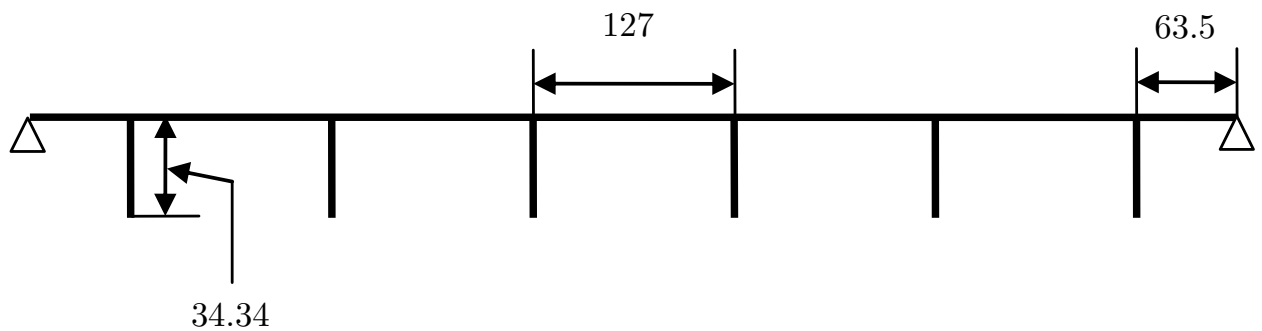

Fig. 16: Cross-section of the "NASA Example 1" panel. Centreline dimensions in $\mathrm{mm}$.

Table 3: Wall construction for the skin and web of the composite blade stiffened panel.

\begin{tabular}{ccccc}
\hline \multirow{2}{*}{ Layer No. } & \multicolumn{2}{c}{ Skin } & \multicolumn{2}{c}{ Stiffener } \\
& Thickness, mm & Fibre orientation & Thickness, mm & Fibre orientation \\
\hline 1 & 0.1397 & 45 & 0.1397 & 45 \\
2 & 0.1397 & -45 & 0.1397 & -45 \\
3 & 0.1397 & -45 & 0.1397 & -45 \\
4 & 0.1397 & 45 & 0.1397 & 45 \\
5 & 0.1397 & 0 & 0.1397 & 0 \\
6 & 1.2573 & 90 & 1.2573 & 90 \\
\hline
\end{tabular}

A comparison of the Abaqus results, as shown in Table 4 to those from VIPASA (single longitudinal wavelength results) shows good correlation, with both buckling load - as calculated from the Abaqus model from the buckling displacement - and order of criticality; with a couple of interesting results. While VIPASA identifies the critical buckling mode to be an $m=8$ mode, Abaqus calculates an $m=9$ mode to be critical, shown in Figure 17. However, this difference of critical mode shape represents only a small variation of buckling load.

Using VIPASA, the mode shape obtained (for the skin) is shown is shown in Figure 18a this shows a buckling half wavelength of $\lambda_{x}=l / 8$ and shows the mode to be skewed. $\frac{18}{18}$ This is due to the small amount of coupling between out-of-plane bending and twisting in the composite stiffened panel, where the effect of the coupling is to produce a skewed mode.

Because the VIPASA mode is skewed, it does not correctly model a periodically supported panel, and as such, it is necessary to use the VICON option in VICONOPT to compute the buckling loads accurately. The equivalent VICON mode shown in Figure $18 \mathrm{~b}$ has no out of plane skin displacement due to the use of point supports on the boundary, these are denoted by crosses in the diagram.

As with the VIPASA results, VICON shows the $m=8$ mode to be critical $(103.763 \mathrm{kN})$, though the buckling load for the $m=9$ mode is very similar 
Table 4: Comparison of Abaqus quad half-bay model buckling results to VIPASA.

\begin{tabular}{cccccc}
\hline Mode No. & \multicolumn{2}{c}{ VIPASA } & \multicolumn{3}{c}{ Abaqus } \\
$m=$ & $\begin{array}{c}\text { Order of } \\
\text { Criticality }\end{array}$ & $\begin{array}{c}\text { Buckling } \\
\text { Load Factor }\end{array}$ & $\begin{array}{c}\text { Order of } \\
\text { Criticality }\end{array}$ & $\begin{array}{c}\text { Buckling } \\
\text { Load Factor }\end{array}$ \\
\hline 9 & 2 & 1.00329 & 1 & 1.00156 & $(-0.173 \%)$ \\
8 & 1 & 1.00000 & 2 & 1.00366 & $(+0.367 \%)$ \\
7 & 5 & 1.02142 & 12 & 1.03171 & $(+1.007 \%)$ \\
6 & 14 & 1.07772 & 43 & 1.09512 & $(+1.615 \%)$ \\
5 & 21 & 1.18655 & 76 & 1.21052 & $(+2.020 \%)$ \\
4 & 28 & 1.38032 & 108 & 1.40733 & $(+1.957 \%)$ \\
3 & 34 & 1.72527 & 148 & 1.74655 & $(+1.233 \%)$ \\
2 & 40 & 2.39882 & 200 & 2.44280 & $(+1.833 \%)$ \\
1 & 19 & 1.15789 & 55 & 1.12388 & $(-2.938 \%)$ \\
\hline
\end{tabular}

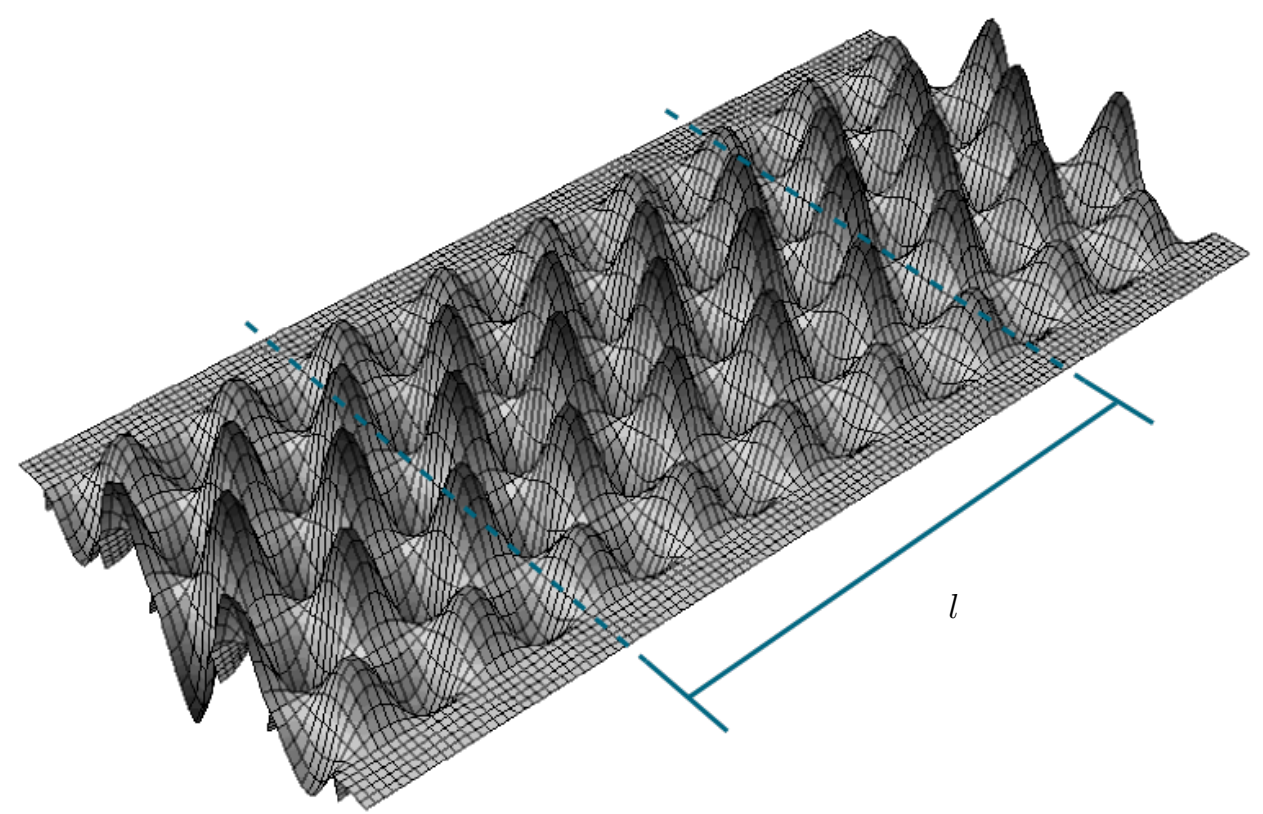

Fig. 17: Critical $(m=9)$ buckling mode of the modified NASA Example 1 panel, quad half-bay model.

$(104.101 \mathrm{kN})$. As VICON uses a combined, multi-wavelength response, identifying mode shapes by wavelength is not truly accurate however, the critical load factors are shown for the first two buckling modes, which are visually equivalent to single wavelength modes.

For Abaqus, the critical $m=9$ mode has a buckling load of $103.909 \mathrm{kN}(1360 \mu \epsilon)$ - as calculated from the modelled buckling displacement — and the $m=8$ mode occurs at $104.128 \mathrm{kN}(1362 \mu \epsilon)$. Both are within $0.4 \%$ error of the VICON equivalent 


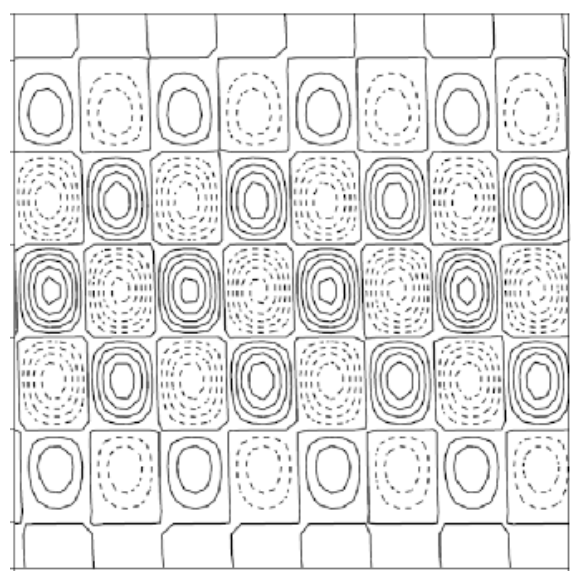

(a) VIPASA mode for $\lambda_{x}=l / 8$

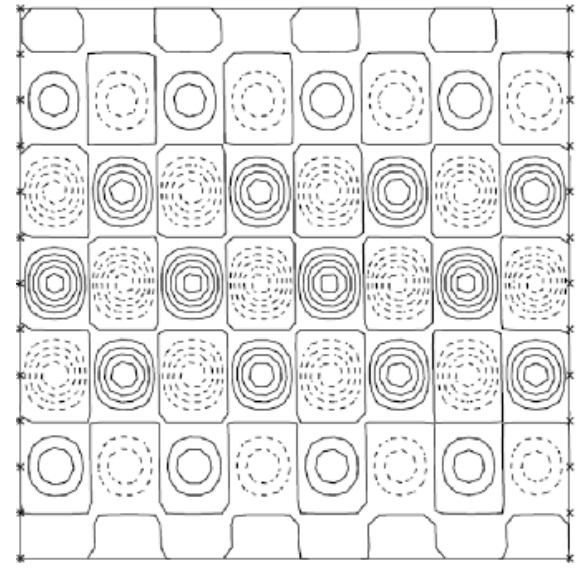

(b) VICON mode shape for $\xi=1, q=5$ in Equation 1.5 .

Fig. 18: Comparison of the skin contour plots for modified NASA Example 1 composite panel where the longitudinal loading direction is left-right.

mode (see Table 5), and within $0.4 \%$ of the critical VICON calculated mode; even though the mode order is different. All the chosen modes are within $3 \%$ of the VICONOPT results. The largest difference between results is shown for the $m=1$ mode that, like with the Example 4 panel, shows a transverse half-wavelength equal to stiffener pitch that is superimposed on the overall mode that is not present in the VIPASA results.

Table 5: Comparison of buckling loads by method to VICON.

\begin{tabular}{cccccc}
\hline $\begin{array}{c}\text { Mode No. } \\
m=\end{array}$ & VICON & \multicolumn{2}{c}{$\begin{array}{c}\text { Buckling Load Factor } \\
\text { VIPASA }\end{array}$} & \multicolumn{2}{c}{ Abaqus } \\
\hline 9 & 1.00325 & 1.00314 & $(+0.314 \%)$ & 1.00141 & $(+0.141 \%)$ \\
8 & 1.00000 & 0.99985 & $(-0.015 \%)$ & 1.00351 & $(+0.351 \%)$ \\
\hline
\end{tabular}

As the critical mode shapes were different depending on the modelling method used, both the $m=8$ and $m=9$ modes were used as post-buckling mode seeds; each with distinct results as shown in Figure 19. For the $m=9$ mode, the postbuckling stiffness was stable up to 4.66 times the critical displacement, staying in the $l / 9$ mode until that point, where it jumped into an overall, $m=1$ collapse mode. For $m=8$, which was also expected to be a stable postbuckling mode, the Abaqus model jumped into the $m=1$ overall mode almost immediately after buckling.

It's worth noting that all material models assume purely elastic behaviour, with no limit of material proportionality and no fracture limit, although these can be 


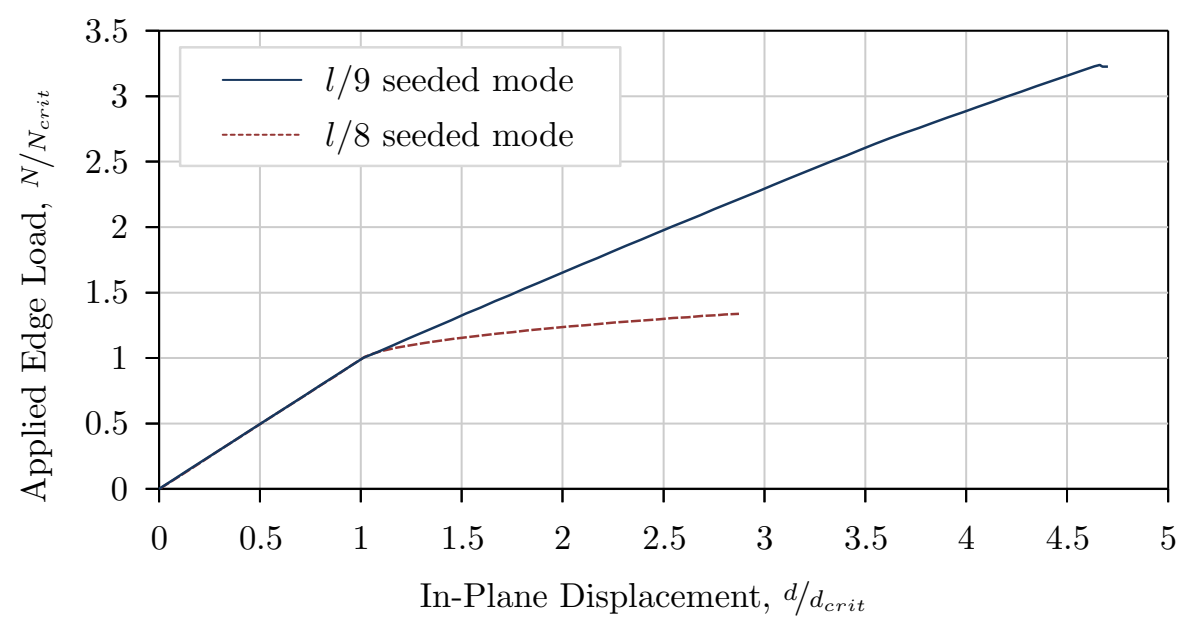

Fig. 19: Force vs. in-plane displacement from Abaqus quad half-bay postbuckling of the modified Example 1 panel.

included in the FEM model.

\section{Conclusion}

The quad half-bay model described in this paper accurately models a periodically supported, infinite length panel where $\lambda / 1$ is the overall mode with the lowest initial buckling load. In addition to the model being logically derived, it has been shown to be accurate compared to VICONOPT's exact finite strip results.

While the FE model identifies each of the modes that VIPASA does, it also gives additional results that are visually identifiable as infinitely repeating modes that do not correspond to a single sinusoidal wavelength. Therefore the FE model finds more valid mode shapes for the infinite length panel than VIPASA.

As well as finding buckling results, the Abaqus model is also suitable for modelling postbuckling performance, having been shown to be comparable to previously published FSM postbuckling models. Each of the postbuckling tests showed mode jumping too, an improvement over previous methods. This postbuckling performance has been displayed for both a homogeneous material and a composite laminate, so it equals the material modelling capability of VICONOPT.

Being able to model infinite length panels using a method other than finite strip theory offers a way of verifying the accuracy of results created by these methods, $\frac{19}{19}$ while including the same assumptions of panel behaviour. 


\section{Acknowledgments}

This work is funded with the help of the Engineering and Physical Sciences Research Council and Airbus UK.

\section{References}

1. Awrejcewicz J., Krysko V.A. and Narkaitis G.G., Bifurcations of a Thin Plate-Strip Excited Transversally and Axially, Nonlinear dynamics, 32, 2003, pp. 187-209.

2. Cheung Y.K., Finite Strip Method in Structural Analysis, Pergamon Press, Oxford, 1976.

3. Zieniewicz O.C., The Finite Element Method, McGraw-Hill, London, 1977.

4. Wittrick W.H., General Sinusoidal Stiffness Matrices for Buckling and Vibration Analysis of Thin Flat-Walled Structures, International Journal of Mechanical Science, Vol. 10, 1968, pp. 949-966.

5. Iyengar N.G.R., Structural Stability of Columns and Plates, Ellis Horwood, 1988.

6. Watson A., Fenner P., Featherston C., Kennedy D., Postbuckling Stability of Panels with Torsional Buckling, 50th AIAA/ASME/ASCE/AHS/ASC Structures, Structural Dynamics, and Materials Conference, 2009, AIAA-2009-2510.

7. Watson, A. and Kennedy, D., Mode Jumping in Post-buckled Stiffened Panels", Proceedings of the International Conference on Thin-walled Structures, Loughborough University, UK, 2004, pp 573-580.

8. Butler, R., and Williams, F. W., Optimum Design Using VICONOPT, a Buckling and Strength Constraint Program for Prismatic Assemblies of Anisotropic Plates, Computers and Structures, Vol. 43, No. 4, 1992, pp. 699-708.

9. Wittrick, W.H., and Williams, F.W., Buckling and Vibration of Anisotropic or Isotropic Plate Assemblies under Combined Loadings, International Journal of Mechanical Sciences, Vol. 16, No. 4, 1974, pp. 209-239.

10. Anderson M.S., Williams F.W. and Wright C.J., Buckling and vibration of any prismatic plate assembly of shear and compression loaded anisotropic plates with an arbitrary supporting structure, International Journal of Mechanical Sciences, Vol. 25, No. 8, 1983, pp. 585-596.

11. Williams F.W. and Anderson M.S., Incorporation of Lagrangian multipliers into an algorithm for finding exact natural frequencies or critical buckling loads, International Journal of Mechanical Sciences, Vol. 25 , No. 8, pp. 579-584, 1983.

12. Wittrick, W.H., and Williams, F.W., An Algorithm for Computing Critical Buckling Loads of Elastic Structures, Journal of Structural Mechanics, Vol. 1, No. 4, 1973. pp. 497-518.

13. Hibbitt, Karlsson, and Sorensen, Inc.,ABAQUS/Standard User's Manual, Version 5.7, 1997, Pawtucket, RI.

14. Stroud W.J., Greene W.H., Anderson MS, Buckling Loads of Stiffened Panels Subjected to Combined Longitudinal Compression and Shear: Results Obtained with PASCO, EAL and STAGS Computer Programs, Computer Methods in Applied Mechanics and Engineering, NASA TP2215, 1984.

15. Dawe D.J., Wang S., Postbuckling Analysis of Composite Laminated Panels, AIAA Journal, Vol. 38, Nov. 2000, No. 11, pp. 2160-2170.

16. Peskham V., Dawe D.J., Buckling and Vibration of Finite-Length Composite Plate Structures with Diaphragm Ends, Part II: Computer Programs and Buckling Applications, Computer Methods in Applied Mechanics and Engineering, Vol. 77, 1989, pp. $227-252$. 
17. Dawe D.J., Lam S.S.E. and Azzizan Z.G., Finite Strip Post-Local-Buckling Analysis of Composite Prismatic Plate Structures, Computers and Structures, Vol. 48, No. 6, 1993, pp. 1011-1023

18. York C.B.,A unified approach to the characterization of coupled composite laminates benchmark configurations and special cases,. Journal of Aerospace Engineering, 23(4), 2010, pp. 219-242.

19. Fenner P., Watson A., Eliminating End Effects for Theoretical Panel Buckling with FEM, 51st AIAA/ASME/ASCE/AHS/ASC Structures, Structural Dynamics, and Materials Conference, 2010, AIAA-2010. 